\title{
Les étudiants «Orientaux » en médecine à Genève (1876-1914)
}

Par Ladislas Mysyrowicz

A la veille de la première guerre mondiale, la Faculté de Médecine de Genève présente, en ce qui concerne son recrutement estudiantin, un contraste marqué avec la situation qui a prévalu depuis les années 20. Cette Faculté est alors la plus cosmopolite, la plus féminine aussi et celle où le militantisme d'extrêmegauche est le plus évident. Ce contraste entre les cinquante premières années et la période ultérieure ne reflète assurément pas une mutation au sein de notre société, car depuis, la Médecine s'est progressivement démocratisée et féminisée. Le tournant de la première guerre mondiale masquerait donc plutôt, sur le plan statistique, cette relative ouverture des études médicales aux femmes et aux couches modestes de notre population. En fait, le changement est dû à une modification radicale dans la proportion et l'origine géographique du contingent étranger, et non à des causes d'ordre sociologique. Avant 1914-1918, le cachet particulier de la Faculté de Médecine de Genève lui était conféré par la masse grandissante des étudiantes et étudiants d'Europe orientale.

Pour comprendre la place occupée par ces étrangers dans la vie de la jeune Faculté, il n'est pas inutile d'indiquer dans quel contexte économique sa création s'est faite. La décision fut prise au lendemain de la guerre franco-allemande et de la Commune, à un moment de grande euphorie financière provoquée par un immense afflux de capitaux français en quête de sécurité ; à quoi s'est ajouté, sur le plan budgétaire, le somptueux legs du duc de Brunswick à la ville. Dans ce climat d'argent facile, un mirage s'est présenté : le projet de fonder une Université fédérale. Pour que notre cité pût se mettre sur les rangs, il lui fallait transformer son Académie en une véritable Université. C'était chose faite en 1876. Mais à cette date, se produit un renversement de conjoncture. Au boom fait soudain place la dépression. Quant au projet d'Université fédérale, il ne se matérialisera jamais. Lorsqu'elle ouvre ses portes, l'avenir de la jeune Faculté ne s'annonce pas sous les meilleurs auspices. Evoquant cette période héroïque, le professeur Laskowski avouait : «J'ai dû commencer ma tâche difficile dans des locaux encore inachevés, sans matériel d'enseignement, sans tradition et presque sans élèves, car ils n'étaient qu'une vingtaine au début. Le corps médical n'était pas très enthousiaste pour la nouvelle création et les contribuables redoutaient de nouvelles dépenses. ${ }^{1}$ Un de ses collègues de la première heure, Jean-Louis Prévost, confiait de même à la fin de sa carrière que «les premiers pas furent durs. Nous nous félicitions de 
pouvoir réunir quelques élèves autour de nous et de professer devant des bancs presque vides ${ }^{2}{ }^{2}$ Comme le soulignait encore un troisième pionnier, l'équipement était insuffisant au début, il n'y avait aucun musée anatomo-pathologique et les recherches étaient difficiles à susciter ${ }^{3}$.

L'étroitesse de la base géographique de la Faculté aggravait ces handicaps. Genève devait par nécessité recruter au dehors, attirer des étrangers sans être magnifiquement dotée ni bénéficier d'une réputation acquise. On misa sur sa position géographique. On espéra en faire un point de rencontre et d'échange entre les courants intellectuels du Nord et ceux de l'Ouest et du Midi. C'est pourquoi, si les chaires de la section dite "professionnelle» furent exclusivement réservées à des praticiens locaux, représentants de l'élite sociale ou personnalités liées aux pouvoirs publics, l'enseignement de base des premières années fut confié à trois professeurs prolongeant en ses trois directions l'espace culturel helvétique: Allemagne (Zahn), France (Laskowski), Italie (Schiff). Mais il s'avéra que les virtualités de ce lieu géométrique ne purent se développer. D’une part, Genève était moins bien soudée à l'espace universitaire germanique que Berne, Bâle ou Zürich et, d'autre part, elle resta séparée de la France et de l'Italie par la rigidité de leurs systèmes académiques: en venant y faire leur Médecine ou y passer quelques semestres, les jeunes Italiens ou Français risquaient de se couper de leurs filières traditionnelles... De manière imprévue, la majeure partie des étudiants arrivèrent de l'Europe de l'Est.

«L'invasion des Orientaux» fut progressive. En sa dixième année d'existence, voici quelle était la situation statistique. L'Université comptait au total 340 inscrits. Près de la moitié, 154, étaient des étrangers. Les Genevois étaient au nombre de 78, tous de sexe masculin: 2 en Théologie, 9 en Droit, 21 en Lettres, 21 en Sciences et 25 en Médecine. Quelques Genevois étudiaient sans doute hors du Canton, mais nous ne les connaissons pas. Le contingent des Confédérés s'élevait à 108, tous également de sexe masculin : 1 en Théologie, 9 en Droit, 19 en Lettres, 25 en Sciences et 54 en Médecine. Parmi les étrangers, 21 nationalités étaient représentées, dont plusieurs sans existence juridique valable - Moravie, Pologne, Macédoine, Arménie, Roumélie Orientale, Géorgie. Ce phénomène a valeur d'indice. Il traduit de la part des intellectuels originaires de ces pays en lutte pour leur autonomie nationale la volonté de s'afficher comme tels et, de la part des autorités locales, l'admission de ces appellations est un des nombreux signes de leur tolérance politique. Quant aux nationalités «Occidentales», elles ne comptaient chacune que quelques rares représentants. L'écrasante majorité des étrangers était d'origine «Orientale». Ce qualificatif amalgame tous les ressortissants de l'Est et du Sud-est européen. La plupart, en Médecine surtout, étaient 
sujets de l'empire russe. Ils appartenaient en règle générale à la catégorie des allogènes : Juifs, Polonais, Géorgiens, Arméniens... Autour d'eux gravitaient des Balkaniques ou des frères de race sujets tures ou persans. En 1886, en Médecine, leur contingent n'est pas encore important : 26 en tout, dont 9 femmes.

Dix ans plus tard, l'Université a presque doublé ses effectifs. Cette progression est essentiellement redevable aux étrangers. A cette date, la Faculté de Médecine est, avec ses 243 étudiants réguliers, la plus importante de l'Université. Près de la moitié d'entre eux appartient au groupe des «Orientaux». Sur ces 117 «Orientaux», on remarque 73 femmes. La Faculté de Médecine de Genève possède dorénavant un "type», qui s'accentuera encore jusqu'à la première guerre mondiale. Les «Orientaux» y viendront de plus en plus nombreux et parmi eux, la proportion des femmes grossira, en dépit de mesures administratives prises pour freiner cet afflux, jugé indésirable par les autorités.

Au début du $\mathrm{XX}^{\mathrm{e}}$ siècle, on observe un ralentissement de la croissance de l'effectif des Suisses. Au semestre d'hiver 1895/96, il y avait 49 Genevois inscrits en Médecine; il n'en reste que $31 \mathrm{dix}$ ans plus tard. Pendant le même laps de temps, le chiffre des autres Confédérés tombe de 65 à 50 inscriptions. Le marché régional de l'emploi, dans cette profession, semble légèrement saturé et de meilleurs débouchés doivent se présenter en Droit et en Sciences, avec le développement des carrières administratives et techniques qu'entraîne dans la structure socio-professionnelle ce qu'on a coutume d'appeler la «seconde révolution industrielle». Si la Faculté de Médecine continue tout de même à progresser numériquement, c'est encore sous la poussée des «Orientaux». Au moment où elle fête ses trente ans d'existence, ils forment les deux tiers de la population estudiantine, soit 200 individus, dont 150 femmes, alors qu'on ne remarque qu'une seule candidate d'origine helvétique, la Genevoise Catherine Humbert. Prenons encore le semestre d'hiver 1911/12. L'Université enregistre alors 1515 étudiants réguliers. La Médecine continue à être la Faculté la plus fréquentée puisqu'elle compte 659 inscrits. Or sur ce chiffre, il n'y a pas moins de 408 Russes, dont trois quarts de femmes, et par dessus le marché, 73 Bulgares. En face, figurent 41 Genevois, 47 Confédérés et un maigre groupe d'«Occidentaux»: 2 Anglais, 4 Italiens, 6 Allemands, 7 Français, 12 Brésiliens, mais aucun Américain du Nord, aucun Belge, ni Espagnol ou Scandinave.

Comment en était-on arrivé à cette disproportion? A quoi attribuer cette «orientalisation» et cette «féminisation» de la Faculté entre 1876 et la première guerre mondiale? Il faut y voir le résultat d'un mouvement migratoire, précédé et accompagné d'un exode politique, tous deux dépassant largement le cadre local. A la fin des années 70, l'agitation révolutionnaire en Russie et la répression poli- 
cière entraînent des dizaines de jeunes intellectuels sur les chemins de l'exil. Genève devient leur principal refuge. Les représentants de tous les courants d'opposition et de toutes les nationalités sujettes s'y donnent en quelque sorte rendez-vous, $\mathrm{y}$ fondent des journaux, des sociétés de secours, des bibliothèques, des cantines. Ces proscrits fréquentent l'Université et s'inscrivent de préférence en Médecine, afin de pouvoir se mettre plus tard au service du peuple. L'immatriculation leur donne automatiquement le droit de séjourner dans le Canton ainsi qu'un petit terrain d'action où faire de vive voix du prosélytisme. En 1887, un correspondant de la Tribune de Genève pouvait ironiser sur le sort de ces Danton et de ces Robespierre réduits à endoctriner de pitoyables auditoires "composés de très petits jeunes étudiants tout naïfs » et, par manque d'une vraie tribune, obligés d'«exercer leur éloquence dans de tout petits cafés ». ${ }^{4}$

A Genève, ces proscrits bénéficient de la connivence des professeurs Laskowski et Schiff. Ouvrons ici une parenthèse pour illustrer cette rencontre.

Sigismond Laskowski fut le premier d'une série d'exilés polonais à jouer un rôle à la fois politique et scientifique au sein du monde universitaire suisse, à la fin du $\mathrm{XIX}^{\mathrm{e}}$ siècle. Il avait participé à l'insurrection de 1863, y avait occupé un poste de responsabilité puis s'était réfugié en France, dont il avait acquis la nationalité. De Genève, Laskowski déploya une activité semi-conspirative en faveur de la renaissance nationale de son pays. Il était lié à Sigismond Balicki, fondateur d'une importante société secrète groupant la jeunesse estudiantine polonaise, et il prit souvent la parole en public pour défendre la cause de sa patrie ${ }^{5}$.

Maurice Schiff, quant à lui, était un ancien révolutionnaire de 1848, comme son ami Carl Vogt. Toute sa vie, il resta fidèle à son idéal de jeunesse. En juillet 1882, par exemple, on le remarque au premier rang d'un important meeting organisé en ville à la mémoire de Garibaldi. Il s'agissait en réalité d'une manifestation antitsariste, placée sous la présidence honoraire du Comité exécutif de la Narodnaja Volja, le groupe terroriste qui venait de s'illustrer en exécutant l'année précédente le tsar Alexandre II. Rien n'était plus mal vu par les Genevois que ce genre de démonstrations «qui font croire au dehors à une perpétuelle agitation dans nos murs et qui chassent les touristes attirés par la beauté de notre lac plus sûrement que ne le ferait la peste ou toute autre maladie contagieuse $»{ }^{6}$ En 1893 se tient à Genève le II $^{\mathrm{e}}$ congrès international des étudiants socialistes. L'organisateur en est un étudiant de la Faculté, Christian Rakowski, qui deviendra par la suite une sommité de la III $^{\mathrm{e}}$ Internationale. Les délibérations sont suivies par de nombreux étudiants et étudiantes d'Europe orientale. Dans la salle drapée de rouge et ornée des portraits des pères du socialisme, voilà qu'apparaît le professeur Schiff. On l'acclame, il monte à la tribune, y évoque son passé de quarante-huitard, les 
combats sur les barricades et lance en conclusion un vibrant appel à la jeunesse universitaire, appelée à diffuser le socialisme dans les masses ${ }^{7}$.

Cette parenthèse nous amène à l'époque où les étudiants russes sont devenus si nombreux qu'ils submergent en quelque sorte la Faculté. La grande majorité d'entre eux était d'origine juive. En Russie, un numerus clausus contingentait leur entrée à l'Université. Depuis 1880, leur quota s'élevait à $3 \%$ des places dans la capitale et à Moscou, et à $10 \%$ dans des villes comme Odessa. Même si ces plafonds ne furent pas toujours strictement observés, il n'en demeure pas moins que d'innombrables candidats étaient exclus des Ecoles supérieures. Ceux qui le pouvaient se rendaient à l'étranger pour y étudier quand même. Ils y vivaient souvent dans une grande misère. On estime qu'à la veille de la grande guerre, 7000 à 8000 sujets russes faisaient leurs études en Europe occidentale et que $90 \%$ d'entre eux étaient de confession israélite. Les Universités allemandes en recevaient le plus grand nombre. Au début du $\mathrm{XX}^{\mathrm{e}}$ siècle, elles commencèrent à prendre tour à tour des mesures discriminatoires contre eux, de sorte que le flot se répandit ailleurs ${ }^{8}$. Mais Genève se distinguait par son activisme politique, plus intense qu'ailleurs, semble-t-il, et par une forte concentration d'étudiantes en Médecine.

Si le militantisme politique de ces étudiants «Orientaux» s'explique assez bien par leur origine, par leur haine contre le régime tsariste, par les persécutions subies chez eux et par le puissant encadrement idéologique qu'ils trouvaient à Genève, la présence d'un pourcentage si élevé de jeunes filles en Médecine n'est pas facile à justifier. Certes, la carrière médicale est à dominante féminine en URSS; nous savons que c'est autant le résultat d'une politique culturelle remontant à 1917 que l'aboutissement d'un mouvement d'émancipation apparu dans les années 18601870 et lié au populisme. A l'époque de la première Internationale, les premières étudiantes en Médecine s'étaient concentrées à Zürich. Elles avaient été ensuite obligées de se disperser ${ }^{9}$. Berne fut leur second lieu de prédilection, puis ce fut Genève, on ne sait trop pourquoi. Des facteurs accidentels, qui nous échappent, ont certainement joué, jusqu'à ce que le mouvement eût pris sa dynamique par une sorte de réaction en chaîne.

La présence massive de ces étudiants qui manifestaient publiquement leur haine envers le régime tsariste et qui affichaient leurs convictions socialistes a constitué un élément important de la vie de la jeune Faculté de Médecine. Mais surtout, ces jeunes gens et ces jeunes filles, rentrés chez eux, ont souvent été des agents de diffusion des doctrines dont ils s'étaient imprégnés au cours de leurs années d'exil. L'écrivain genevois Philippe Monnier l'avait bien compris. «Quelqu'un qui habite la Russie, écrivait-il en 1905, m'apprenait dernièrement qu'il y 
a à la campagne des populations entières gagnées aux idées nouvelles. Il attribuait cette attitude imprévue aux leçons des instituteurs, esprits souvent très distingués, éloignés des villes, relégués au fond des provinces par les soins du gouvernement. Ces instituteurs ont dû trouver des collaboratrices précieuses dans les anciennes élèves de Maurice Schiff et de Carl Vogt. »10

\section{Notes}

${ }^{1}$ Manifestation en l'honneur de Monsieur le professeur S.-L.Laskowski, 29 juin 1916, s.l.n.d.

${ }^{2}$ Manifestation en l'honneur de Monsieur le professeur Jean-Louis Prévost, 27 juin 1913, s.l.n.d.

${ }^{3}$ Zahn, F. Wilh., Les vingt-cinq premières années de l'Institut pathologique de Genève, Genève 1901, p.5-7.

4 Tribune de Genève, 7 avril 1887.

${ }^{5}$ Quelques documents d'archives (AEG) et la chronique locale de la presse fournissent des indications sur son activité politique, ainsi que la monographie de Janusz Pajewski, Wokól sprawy polskiej, Poznan 1970.

${ }^{6}$ Les comptes rendus de presse sont muets sur la teneur du discours prononcé à cette occasion par le professeur Schiff, en compagnie d'orateurs tels qu'Eduard Bernstein, Ludovico Nabruzzi, Louis Héritier, Kaspar Tursky ; cf. la presse genevoise, 1-4 juillet 1882.

${ }^{7}$ Cf. la presse genevoise, décembre 1893-janvier 1894. - Le 28 janvier 1893, le Conseil universitaire des étudiants portant couleurs organisait un cortège aux flambeaux en l'honneur du professeur Schiff. Un étudiant russe, Micislas Certowitch, s'y joignit inopinément et félicita le maître au nom des étudiants socialistes de l'Université (cf. Journal de Genève, 29 et 31 janvier 1893).

${ }^{8}$ Vucinich, Alexander, Science in Russian Culture, II, 1861-1917, Stanford 1970, p. 485.

${ }^{9}$ Meijer, Jean M., Knowledge and Revolution. The Russian colony in Zurich. Assen 1955.

${ }^{10}$ Gazette de Lausanne, 26 janvier 1905.

\section{Summary}

This article examines the student population of the Medical Faculty between 1876 and 1914 . It pays special attention to the numerical preponderance of male and female students coming from Eastern European countries. It reports, on the basis of documents, the intensive revolutionary activities of this period.

\section{Ladislas Mysyrowicz}

14a, avenue Ernest Pictet

1203 Genève 